CARNETS DE Carnets de géographes

GÉOGRAPHES.

$7 \mid 2014$

Les espaces de l'entre-deux

\title{
A demeure en exil ? Etre réfugié tamoul sri lankais au Tamil Nadu
}

Anthony Goreau Ponceaud

\section{(2) OpenEdition}

\section{Journals}

Édition électronique

URL : http://journals.openedition.org/cdg/418

DOI : $10.4000 /$ cdg. 418

ISSN : 2107-7266

Éditeur

UMR 245 - CESSMA

Référence électronique

Anthony Goreau Ponceaud, «A demeure en exil ? Etre réfugié tamoul sri lankais au Tamil Nadu », Carnets de géographes [En ligne], 7 | 2014, mis en ligne le 01 décembre 2014, consulté le 30 avril 2019. URL : http://journals.openedition.org/cdg/418 ; DOI : 10.4000/cdg.418

La revue Carnets de géographes est mise à disposition selon les termes de la Licence Creative Commons Attribution - Pas d'Utilisation Commerciale - Pas de Modification 4.0 International. 


\title{
A DEMEURE EN EXIL? \\ ETRE REFUGIE TAMOUL SRI LANKAIS AU TAMIL NADU
}

\author{
ANTHONY GOREAU-PONCEAUD \\ Géographe \\ Université de Bordeaux \\ UMR 5185 ADESS CNRS \\ anthonygoreau@yahoo.fr
}

\section{Résumé}

Cet article porte sur les migrants tamouls sri-lankais vivant en camp au Tamil Nadu depuis les années 1980. Fondé sur des données collectées au gré de plusieurs séjours entre 2010 et 2013, il explore la question de la marginalisation, des limitations et des processus d'exclusion dont souffre cette population, tout en nuançant ce constat en mettant l'emphase sur la manière dont les pratiques et les initiatives de ces migrants infléchissent ces processus de mise à l'écart en produisant des espaces de l'entre-deux.

\begin{abstract}
This article deals with the Sri Lankan Tamils refugees in Tamil Nadu, from the first arrivals in 1983, following the Sinhalese violence against the Tamils after black July pogrom up to the present days. Based on both primary and secondary data collected through a filed survey conducted in June 2010, June 2012 and July and August 2013, this article explore the issue of marginalization, limitation and exclusion processes that affected the Sri Lankan refugees in Tamil Nadu. By using interviews in order to reveal their life story, this article shows that the practices of these migrants produce in-between spaces.
\end{abstract}




\section{Introduction}

Voici : lorsqu'un nageur courageux traverse un fleuve large ou un détroit éventé, l'itinéraire de son voyage se divise en trois parties. Aussi longtemps qu'il garde en vue la rive du départ ou découvre celle d'arrivée, il habite encore son gîte d'origine ou déjà le but de son désir [...] Or, vers le beau milieu du parcours, vient un moment, décisif et pathétique, où à égale distance des deux rivages, pendant le passage, plus ou moins durable, d'une grande bande neutre ou blanche, il n'est encore ni l'un ni l'autre et, devient peut-être, déjà, l'un et l'autre, à la fois. Inquiet, suspendu, comme en équilibre dans son mouvement, il reconnaît un espace inexploré, absent de toutes les cartes et qu'atlas ni voyageur ne décrivirent (Serres, 1997 : 24).

Port de Pondichéry, vendredi 2 août 2013, 9h40, il fait chaud, déjà très chaud, $32^{\circ} \mathrm{c}$ et $76 \%$ d'humidité. Un premier pick-up portant son chargement d'hommes, de travailleurs sans contrat arrive enfin à destination (Figure 1). Il sera suivi par six autres engins du même type et d'un camion, apportant finalement un total de 731 travailleurs : tous des hommes, tous Tamouls, tous originaires du Nord Sri Lanka, tous ayant fui la guerre pour trouver refuge et sécurité au Tamil Nadu. Les voitures ne repartiront que tard dans la nuit, vers $23 \mathrm{~h} 50$ pour ramener ces travailleurs "chez-eux», au camp de Keezhputhupattu dans le district de Villupuram, à une vingtaine de kilomètres au Nord de Pondichéry le long de la fameuse " scenic beach way " comme l'aime à le rappeler le gouvernement du Tamil Nadu, dans un souci de développement touristique : la East Coast Road (ECR). Ces scènes visibles au port de Pondichéry se répètent également depuis deux mois dans le district de Cuddalore, plus au Sud, où les habitants des camps de Kurinjipadi et Vriddhachalam affluent dans le port de Cuddalore pour prêter main forte. II faut dire que c'est la pleine saison de pêche sur la côte de Coromandel. De juin à octobre, les activités halieutiques sont intenses, et les camions des Etats voisins - Kerala et Karnataka - où la pêche est arrêtée du fait de la mousson, attendent avec impatience les poissons pour y être expédiés. Les plus gros et les moins appréciés localement, thon rouge et barracuda en particulier, partiront via le Kerala au Japon. C'est tout un secteur d'activité qui a besoin, pour un temps, d'une main-d'œuvre importante, disponible et peu revendicative. Le port de Pondichéry prend durant cette saison les allures d'un espace de transaction où pour un temps seulement, une population en marge devient visible, de sorte qu'il se joue ici quotidiennement ce que Michel Lussault nomme une "lutte des places " (2009), où le devenir visible passe par la matérialité et donc la corporéité de ces travailleurs.

Ces « espaces écarts » (Doraï et Puig, 2012 : 19), à l'instar du camp de Keezhputhupattu qui ont été introduits pour les contrôler, racontent pourtant les histoires corporelles de ce que sont les conditions préalables à l'accès à une forme de citoyenneté. Ces espaces procèdent non pas d'une distinction entre Indien et Sri Lankais mais d'une distance permettant ce que Jullien nomme un dévisagement réciproque de l'un par l'autre : où l'un se découvre lui-même en regard de l'autre, à partir de l'autre, se séparant de lui (2012: 7). Il s'agit d'espaces de mise en tension de l'un et l'autre, de l'inclus et de l'exclu qui donnent à penser l'entre-deux, entre I'(en)placement et le (dé)placement.

En août 2013, il y avait au Tamil Nadu 117 camps accueillant une population dépassant les 73000 personnes. Ces autres sociaux, à demeure en exil, entre deux ailleurs et deux absences, ne sont ni citoyens indiens, ni réfugiés, ni totalement inclus, ni totalement exclus : 
ils échappent à l'ensemble des conceptions binaires qui colorent nos imaginaires et rhétoriques politiques actuelles. Rappelons comme le note Nuselovici (2013: 5) que l'expérience exilique conjoint lieu de départ et lieu d'arrivée (...) suscitant et étayant une dynamique de multi-appartenance dont les logiques citoyennes des Etats-nations ne parviennent pas toujours à intégrer la complexité.

Née en 1947 d'une partition qui a généré quatorze millions de réfugiés, l'Inde indépendante ne reconnaît pas pour autant le statut juridique international de réfugié : elle n'a pas ratifié la Convention de Genève de 1951 et le protocole de 1967 et elle n'a pas élaboré de définition propre à ce statut à travers une loi nationale (Samaddar, 2003 ; Baujard, 2012 ; Oberoi, 2006). L'Union indienne traite les réfugiés entrant sur son territoire, en fonction de leur origine nationale et selon des considérations politiques, laissant la question de l'égalité et de l'uniformité des droits et privilèges accordés aux différentes communautés de réfugiés ${ }^{1}$ sans réponse. $L^{\prime}$ Inde conserve ainsi sa pleine souveraineté dans la gestion des réfugiés (Samaddar, 2003). Ce statut de réfugié, a priori défini par des institutions nationales ou internationales, selon des critères normatifs et juridiques, ne l'est donc pas dans le cas indien. Pourtant, ces Sri Lankais sont bien des réfugiés, administrés directement par l'Inde, plus particulièrement par le Tamil Nadu, sans que l'agence des Nations Unies n'intervienne sauf dans le cas particulier des opérations de "rapatriement ${ }^{2}$. Combiné avec leurs trajectoires souvent précaires, ce non-statut les expose à de multiples restrictions quant au logement, à l'accès au marché du travail et à la liberté de mouvement. Ils sont soumis à des dynamiques juridiques, économiques, politiques et sociales qui consolident leur altérité.

Cependant, en m'appuyant sur des enquêtes menées au Tamil Nadu en juin 2010, juin 2012 et juillet-août $2013^{3}$, je voudrais montrer que les pratiques et les initiatives de ces migrants infléchissent ces processus de marginalisation, de limitation et d'exclusion. Ces enquêtes ont

\footnotetext{
${ }^{1}$ Afghans, Bengalis, Bhoutanais, Birmans, Chakmas, Népalais, Sri Lankais et Tibétains principalement.

${ }^{2}$ Le gouvernement indien a accordé au HCR un mandat limité. Le HCR a en effet la charge des réfugiés urbains non sud-asiatiques, ce terme ayant pour le gouvernement une forte connotation géopolitique : le HCR assurant par exemple la protection des Afghans et des Birmans mais pas celle des Tibétains (Baujard 2012 et Sen 2003). Le HCR n'a pas accès aux camps de réfugiés. Néanmoins, en vertu d'un accord de juillet 1992 passé avec le gouvernement indien, le HCR a été invité à aider le gouvernement à déterminer la nature volontaire du rapatriement de Sri Lankais dans leur pays. En réponse à la demande, le HCR a obtenu l'autorisation d'ouvrir un bureau à Chennai en août 1992 (communication personnelle avec Vidjea Bharaty, officier général de rapatriement, Besant Nagar, juin 2012, HCR de Chennai).

${ }^{3}$ Lors de mon doctorat de géographie portant sur la diaspora tamoule (Goreau-Ponceaud, 2008) j'avais pu mener quelques entretiens au camp de transit de Mandapam au sein duquel s'élaboraient des stratégies quant aux routes migratoires vers l'Europe. Ce n'est qu'en 2010 que j'ai pu retourner au Tamil Nadu et mener une réflexion plus approfondie sur les diverses politiques adoptées et mises en œuvre par le gouvernement indien envers les réfugiés sri-lankais. L'accès au camp à toute personne extérieure étant interdit, même avec un visa de recherche, je me suis dans un premier temps tourné vers les ONG autorisées à intervenir dans les camps. C'est dans ce cadre que j'ai mené divers entretiens à Chennai auprès des membres de l'ONG Organisation For Eelam Refugees Rehabilitation (OfERR) et que j'ai sympathisé avec Sugumaran, le secrétaire général de Federation for People's Rights (FPR), une ONG située à Pondichéry. L'ONG OfeRR m'a donné accès à l'ensemble des statistiques dont elle dispose. Grâce aux relations de Sugumaran j'ai pu mener mes premiers entretiens au camp de Keezhputhupattu et revenir au camp de Mandapam. Néanmoins, je n'ai pu pénétrer que deux fois dans le camp de Keezhputhupattu. La majorité des entretiens se déroulait en dehors du camp. Lors de ce terrain j'ai noué une amitié avec Ruban Kuroos, un réfugié originaire de Mannar, arrivé au Tamil Nadu en 1992. C'est grâce à lui que j'ai pu découvrir la variété des espaces investis par les réfugiés et que j'ai commencé à mener mes entretiens au port de Pondichéry, durant la saison de pêche. Je tiens à remercier ici l'UMR ADESS et I'Institut Français de Pondichéry (IFP) pour leur soutien dans cette recherche.
} 
été rendues possibles par la collaboration d'un assistant de recherche. Les entretiens se faisaient en tamoul et leur retranscription en anglais, puis en français. Bien entendu, je suis conscient des effets de la traduction/transcription/interprétation sur l'information contenue dans les entretiens. Par leur façon de prendre place, les Sri Lankais composent des territoires selon des temporalités (une saison de pêche par exemple) et des rythmes variables. Ces migrants déploient des ancrages divers développant différentes figures de l'entre-soi. Ces figures de l'entre-soi, en fonction de qui regarde, peuvent être qualifiées tours à tours d'emprise habitante, $d$ ' "espace écart ", de contre-espace, d'espace de l'entre-deux: il s'agit là d'un jeu onomastique complexe pour des populations labiles prises dans ce que Zygmunt Baumann (2010) nomme la " modernité liquide ». Ces espaces permettent de tisser du lien, de prendre place, pour des personnes qui se décrivent elles-mêmes " out of place » ou encore idampeyarntha, c'est-à-dire déplacées.

Après avoir brossé un rapide historique de la présence sri-lankaise au Tamil-Nadu, je montrerai de quelle manière ces espaces de l'entre-deux sont le produit à la fois du contrôle institutionnalisé des frontières et des initiatives et des pratiques des Sri Lankais qui développent des formes de cohabitations inédites. Avec leurs différentes manières de faire, de prendre place, les réfugiés sri-lankais créent dans ces espaces, leurs propres ressources, garantes d'une autre normalité. Pris dans une tension permanente entre formel et informel, reconnaissance et déni, les Sri Lankais modèlent des espaces - véritables interstices permettant leur intégration partielle - dans lesquels ils s'inscrivent dans des relations de pouvoir qui les placent dans un rapport de domination. Analyser ce type d'espace nécessite d'adopter un décentrement, épistémologique et situationnel qui consiste à déplacer le lieu et le moment du regard depuis le centre et l'ordre vers les bords et le désordre (Agier, 2012 : 53).

\section{Figure 1 : Réfugiés Sri Lankais arrivant au port de Pondichéry}

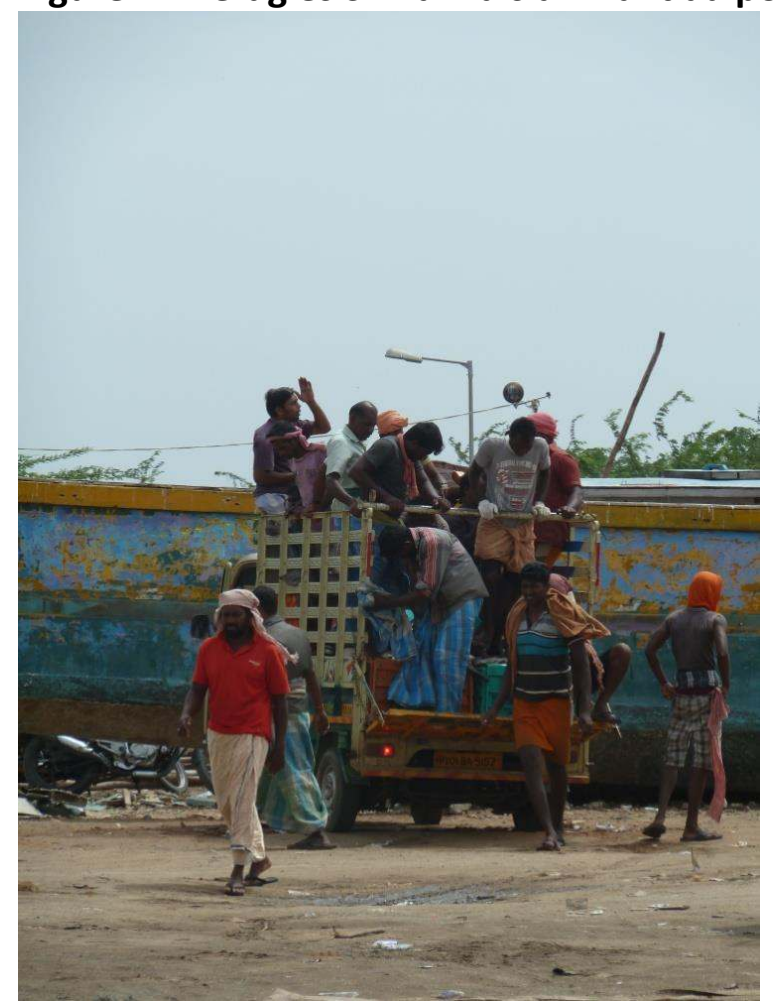

Cliché : Goreau-Ponceaud, août 2013 


\section{Trouver refuge en Inde}

"En raison des politiques discriminatoires successives du gouvernement cinghalais depuis l'indépendance, en ce qui concerne la langue, l'éducation et l'emploi, j'ai perdu ma confiance dans l'État et dans le gouvernement sri-lankais. Les émeutes périodiques anti-Tamouls, la montée du militantisme et le harcèlement mené à la fois par les forces armées sri-lankaises et

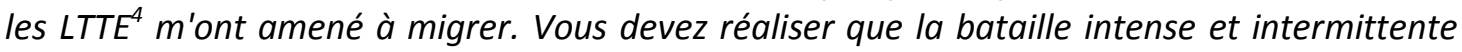
entre les forces armées et les groupes militants tamouls, depuis les années 1980, a transformé le nord-est en une zone de guerre et a mis sur les routes de l'exil un nombre considérable de Tamouls. J'ai donc moi aussi fait le choix de partir. Je suis arrivé au Tamil Nadu en 1990. Je craignais pour ma sécurité et mon intégrité physique. J'ai donc fait ce choix douloureux de quitter Mannar et ma famille. J'ai laissé derrière moi ma mère, mon père et ma sceur. D'ailleurs je ne sais pas s'ils sont vivants ou morts à l'heure où je vous parle. J'ai pris la fuite car j'avais vraiment peur. Une peur telle que lorsque j'entendais des bruits de simples pétards pour une fête, à l'occasion de thaipusam ou deepavali, je me cachais, je croyais qu'il s'agissait d'une attaque. D'une attaque de l'armée ou des Tigres. J'ai donc contacté une " boat agency » et j'ai pris la mer contre 40000 roupies $^{5}$. Mais tu sais, il faut faire attention. II y a trois dangers principaux: l'armée cinghalaise, les LTTE et l'Indian Navy. La traversée a duré 3 heures, mais cela dépend surtout de la puissance du bateau. Je suis arrivé à Rameswaram. Avant d'arriver au camp de Keezhputhupattu, où je suis installé avec ma femme, mes deux garçons et ma fille, on m'a d'abord envoyé au camp de Palar Anaikattu à Vellore. [...] Je ne veux pas retourner à Sri Lanka. Mes enfants ne connaissent que I'Inde. Ils sont nés au Tamil Nadu et de toute façon retourner à Sri Lanka n'est pas sans danger. Rien ne $m^{\prime} y$ pousse à vrai dire. Je sais que mon village a été détruit et que nos terres ont été confisquées par le gouvernement ". (Extrait d'un entretien mené avec Rajan. Port de Pondichéry, 2 août 2013).

"I'ai pris le choix de quitter Sri Lanka en 2009. J'ai pris le bateau pour Rameswaram. La traversée $m^{\prime}$ a coûté 70000 roupies $^{6}$. Au camp de transit de Mandapam on m'a demandé où je voulais me rendre. Je voulais venir à Keezhputhupattu - j'ai des amis ici-mais il n'y avait plus de place. Alors on m'a envoyé à Tiruvannāmalai, au camp d'Athiyandal » (Entretien avec Rajesh, camp de Keezhputhupattu, 20 juin 2010).

" Je m'appelle Udaidinesh, j'ai 27 ans, et je suis né en Inde en 1985, non loin de Vellore. Mes parents ont fait enregistrer ma naissance auprès du Sri Lanka Deputy High Commision à Chennai pour que j'obtienne la citoyenneté sri-lankaise. Je suis citoyen sri-lankais mais je vis en Inde, je réside au camp de Keezhputhupattu. En 1987, à l'âge de deux ans, ma famille est retournée à Sri Lanka. J'ai passé trois années là-bas avant de revenir en Inde en 1990. Tous ces allers et retours c'est à cause de la guerre. La première fois on a pris l'avion et les autres fois toujours le bateau. Il faut dire que c'est plus aisé car nous sommes de Pesalai, sur lîle de Mannar. C'est à moins de $20 \mathrm{~km}$ de Rameswaram. Après le tsunami de 2004, on est retourné à Sri Lanka. Pendant trois années, grâce à l'implication de la Norvège, il y a eu un cessez-lefeu et on a pu vivre normalement, sans craindre pour nos vies, pour notre sécurité. Mais en 2007, tout à recommencé. On a eu très peur. Ma mère, mon père et mes trois sceurs ont été kidnappés. Plus exactement c'est toutes les personnes du même bus qui ont été kidnappées.

\footnotetext{
${ }^{4}$ Les Tigres de libération de l'Eelam tamoul ou LTTE sont un mouvement indépendantiste, fondé en 1976 par Velupillai Prabhakaran, dont le but affiché est de défendre les Tamouls de Sri Lanka. Pour cela, l'organisation demande le droit à l'autodétermination et la création d'un Etat, le Tamil Eelam. La défaite militaire du 17 mai 2009 a ébranlé le mouvement.

${ }^{5}$ Toutes les sommes mentionnées dans le texte sont exprimées en roupies indiennes.

${ }^{6}$ La hausse du prix de la traversée s'explique par la dévaluation entre 1990 et 2009 de la roupie indienne.
} 
Mes parents et mes sœurs ont vu des gens se faire tuer et d'autres subir des atrocités. Des enfants ont été démembrés devant eux. Mes parents n'ont pas été tués car ils avaient leur carte d'identité sri-lankaise sur eux. C'est pourquoi on a fui une fois de plus pour trouver refuge en Inde. Nous ne voulions plus connaître une pareille souffrance nous étions trop effrayés. Ici nous travaillons beaucoup, la vie est très dure mais je ne crains pas pour ma vie. $A$ Sri Lanka je ne pouvais pas dormir, je ne trouvais pas le sommeil. En 2007 on a donc pris la décision de rester, de vivre dans le camp de Keezhputhupattu, ma mère, moi et deux de mes sœurs. La troisième vit en dehors des camps. Elle a un bon travail et habite à Chennai, dans le Sud je crois. Là-bas il y a aussi beaucoup de Tamouls sri-lankais. Elle gagne beaucoup d'argent, mais je ne peux pas lui demander de m'aider. Si c'était un frère, cela serait différent. Mon père lui a décidé de rester à Pesalai. Il continue ses activités de pêcheur mais nous a dit que cela ne valait pas le coup de revenir. Depuis 2009, les Cinghalais sont les rois et dominent même dans la partie tamoule. C'est pourquoi mon souhait c'est de partir en Australie. Mais avant je dois travailler beaucoup pour aider à payer le mariage de mes trois sœurs." (entretien mené le 3 août 2013 au port de Pondichéry).

Le Sri Lanka, île de l'océan indien de plus de 21 millions d'habitants, dont les trois-quarts ont pour langue maternelle le cinghalais (une langue issue du fonds indo-aryen) et le cinquième, le tamoul (langue dravidienne $d^{\prime}$ Inde $d u(S u d)^{7}$, a été marqué pendant près de trois décennies par un conflit séparatiste sanglant, déclenché par des groupes militants appartenant à la minorité tamoule autochtone. Ce conflit a eu de larges répercussions dans les logiques migratoires des Sri Lankais: terre d'immigration depuis les débuts de son histoire, le Sri Lanka est devenu progressivement au cours des années soixante-dix, une terre d'émigration massive. La mise en place de lois discriminantes ("Sinhala only "), a lancé sur les routes de l'exil, de manière sporadique dans un premier temps, des élites anglophones vers les pays du Commonwealth. Néanmoins, après les pogroms anti tamouls de juillet 1983, correspondant également à la montée en puissance du mouvement des Tigres de libération de l'Eelam tamoul, les flux de réfugiés et de demandeurs d'asile en direction des pays du Nord, d'Asie (Malaisie et Inde en particulier) et du Moyen-Orient sont devenus significatifs conduisant à la formation d'une diaspora ${ }^{8}$ et à une diversification des profils migratoires. En 2010, le poids démographique de cette diaspora était estimé a environ 800000 personnes, soit plus du quart de l'ensemble de la population tamoule de l'île, ce qui fait dire à Ruban qu' " à terme les Tamouls seront plus nombreux en dehors de Sri Lanka, et la fin de la guerre n'y changera rien » (entretien, juin 2012).

\footnotetext{
${ }^{7}$ Dans la société sri lankaise se juxtaposent des communautés qui diffèrent par la langue, la religion et les structures sociales. D'origine indo-aryenne (du nord de l'Inde) et à grande majorité bouddhiste, la communauté cinghalaise est la population majoritaire dans le pays (74\%). La population tamoule, d'origine dravidienne (sud de l'Inde) est scindée en deux ensembles : les Tamouls "autochtones" (12,6\%) et les Tamouls des plantations (5\%), qui ont un statut et des intérêts très différents. Les Tamouls "autochtones", présents bien avant la colonisation aux côtés des Cinghalais, ont le sentiment d'être, tout comme ces derniers, des "enfants du sol" et non des immigrés. Les Tamouls des plantations, arrivés au XIXe siècle, ont été utilisés, sous la domination britannique, comme main d'œuvre dans les plantations du centre du pays, enclavées dans des régions à forte majorité cinghalaise.

${ }^{8}$ Dans cette contribution, le terme de diaspora est utilisé comme une catégorie analytique permettant de décrire les processus de migration et les relations transnationales qui lient les Tamouls sri-lankais déplacés entre eux, avec le pays d'origine (ou homeland) et l'ailleurs (œcoumène diasporique). Plus exactement, dans la continuité des travaux de Brubaker il s'agit moins de se demander ce qu'est une diaspora, que d'étudier les sens du terme de diaspora donné par les Tamouls eux-mêmes qui se réclament d'une diaspora. Comme le précise Brubaker, In sum, rather than speak of 'a diaspora' or 'the diaspora' as an entity, a bounded group, an ethnodemographic or ethnocultural fact, it may more fruitful, and certainly more precise, to speak of diasporic stances, projects, claims, idioms, practices, and so on (2005: 13).
} 
Cette diaspora n'est pas un ensemble homogène ${ }^{9}$ et son appartenance n'est pas revendiquée par tous. Les migrants Tamouls, particulièrement ceux installés en Europe, en Amérique du Nord et en Australie se sont bien organisés à travers la création et la multiplication d'associations. Ils se sont ménagés ainsi une "niche de visibilité " dans l'espace public en présentant une " ethnicité homogène " et contribuent par extension au renforcement des liens communautaires et en retour à la structuration de chaînes migratoires (Goreau-Ponceaud, 2014). Mais il existe en miroir de ces centralités diasporiques (l'espace de la diaspora est un cercle dont le centre est partout et la circonférence nulle part), une périphérie constituée par les migrants les plus pauvres (majoritairement agriculteurs et pêcheurs) qui n'ont eu pour autre choix que de migrer et demeurer en Inde. Même si les facteurs "push » restent similaires, les trajectoires sont différentes. En effet, c'est l'expérience quotidienne de l'insécurité, telle qu'elle transparaît dans les récits de Rajan et Udaidinesh, qui permet de comprendre pourquoi des milliers de Tamouls ont quitté leur pays pour un autre plus pauvre. Le schéma pour fuir Sri Lanka reste souvent le même : une des premières étapes, non des moindres, est de traverser le détroit de Palk pour se rendre en Inde. Jusqu'en 2009, la traversée était assurée par les LTTE, ironiquement ${ }^{10}$ appelés "Eelam Shipping Service ". Les migrants voulant fuir le Sri Lanka et les zones de combats utilisaient deux routes principales (Figure 2): la plus usitée était TalaimannarRameswaram, et dans une moindre mesure Nacheguda-Rameswaram.

\footnotetext{
${ }^{9}$ Cette diaspora est marquée par de forts antagonismes reposant entre autres sur une opposition de classe socio-économique, d'origines géographiques des migrants (péninsule de Jaffna dans les années 1980, province du Wanni dans les années post-1990), de castes (les Vellalar des années 1980 face aux Karaiyar des années post-1990), de statut (les immigrés naturalisés des années 1980 arrivant directement de Sri Lanka en famille face aux demandeurs d'asile des années post-1990 majoritairement célibataires), de positionnements idéologiques face aux actions entreprises par les LTTE.

${ }^{10}$ De 1965 à 1983 a existé une liaison ferroviaire entre Colombo et Chennai avec une partie par ferry entre Rameswaram (ou Rameshwaram) et Mannar. En raison de l'escalade de la violence entre l'armée et les forces séparatistes, la liaison a été officiellement interrompue. C'est donc de manière illégale que les LTTE ont mis en place ces liaisons maritimes.
} 


\section{Figure 2 : Routes migratoires et camps de refugiés au Tamil Nadu}

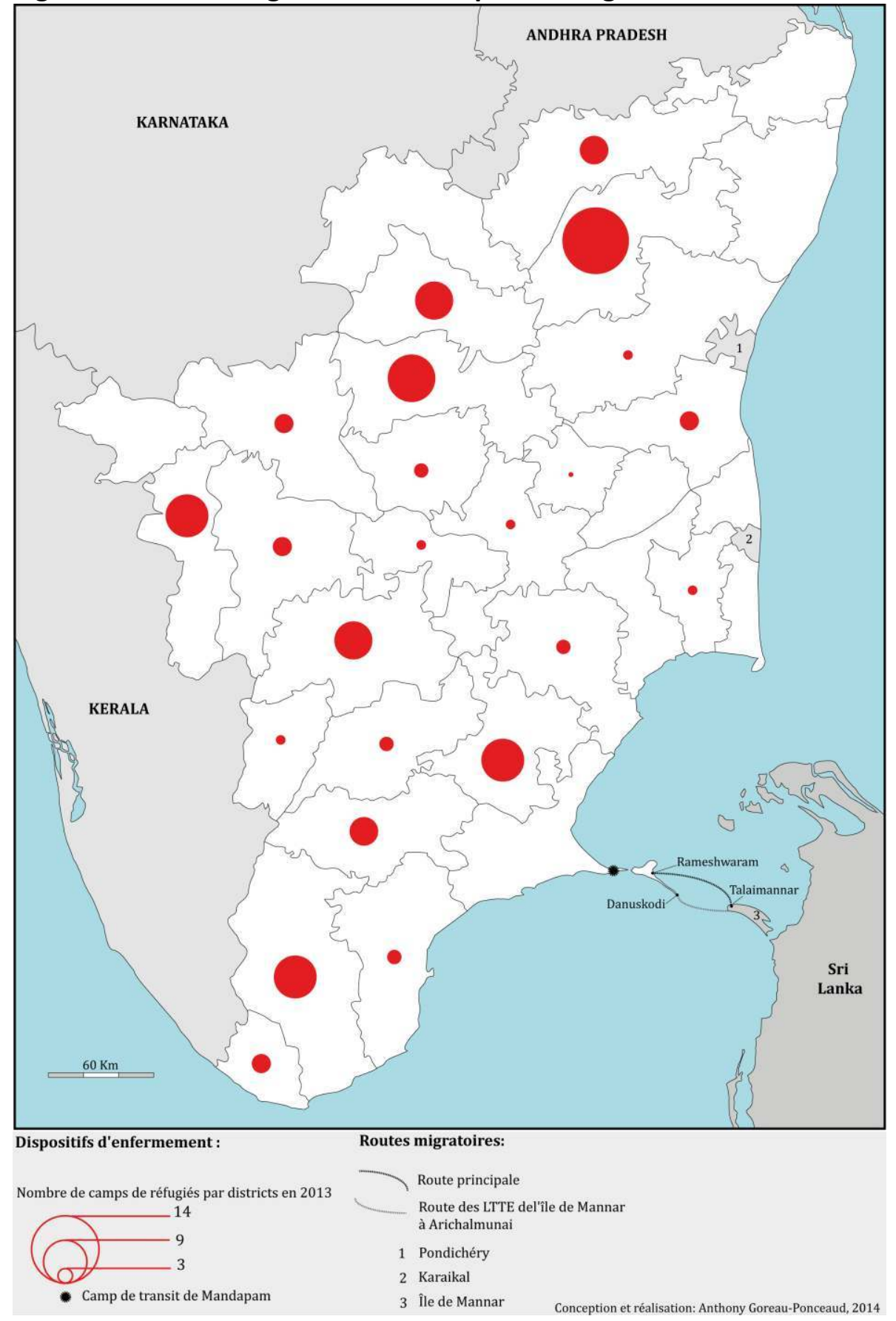

La contiguïté géographique - moins de 16 kilomètres entre l'île de Mannar et Rameswaram - les affinités ethniques et la disponibilité de bateaux font du Tamil Nadu un choix « naturel ${ }^{11}$. Dès le début du conflit, le Tamil Nadu est apparu comme une terre de refuge

\footnotetext{
${ }^{11}$ Au début des années 1980, le gouvernement régional du Tamil Nadu a accordé aux militants du LTTE des facilités pour s'armer et s'entraîner. En dépit des évolutions politiques, il existerait encore des camps d'entraînement dans les districts des Nilgiris et de Coimbatore (Smith, 2008 et entretiens de juin 2010 auprès de Pala Nedumaran). Par ailleurs, il existe une ethnicité commune, entre Tamouls du Sud de l'Inde et Tamouls Sri-lankais (dont les ingrédients sont l'hindouisme shivaïte, le culte de Murugan et la langue) construite comme un impératif politique permettant la sauvegarde de l'identité tamoule face à la domination, d'une part du
} 
pour les Tamouls sri-lankais (figure 3); et le flot de réfugiés est dépendant des évolutions politiques de l'île (Suryanarayan, 2000 ; Oberoi, 2006), ce que montre le récit d'Udaidinesh.

Figure 3 : Flux de réfugiés Tamouls sri-lankais

\begin{tabular}{|l|l|l|}
\hline Phase & Période & Nombre de réfugiés \\
\hline Guerre d'Eelam 1 & $1983-1987$ & 134053 \\
\hline Guerre d'Eelam 2 & $1990-1995$ & 122078 \\
\hline Guerre d'Eelam 3 & $1995-2002$ & 23356 \\
\hline Guerre d'Eelam 4 & $2006-2009$ & 24527 \\
\hline Total & $\mathbf{3 0 3 0 7 6}$ \\
\hline
\end{tabular}

Department of rehabilitation, Chennai, juin 2012.

En effet, le premier exode a commencé peu après le juillet noir en 1983 et a continué jusqu'en juillet 1987, date de l'intervention de I'Indian Peace Keeping Force $e^{12}$ dans le conflit sri-lankais, lorsque un accord Inde-Sri Lanka a été signé (Singh, 2003 ; Dasgupta, 2003). Durant cette période, 134053 Tamouls sri-lankais trouvent refuge en Inde du Sud. Suite à l'accord Inde-Sri Lanka, les réfugiés commencent à retourner à Sri Lanka (Figure 4).

Figure 4: Opération de " rapatriement " et nombre de réfugiés retournant à Sri Lanka

\begin{tabular}{|l|l|}
\hline Période & Nombre de réfugiés \\
\hline $1987-1989$ & 25885 \\
\hline $1992-1995$ & 54188 \\
\hline $2003-2005$ & 65000 \\
\hline
\end{tabular}

\begin{tabular}{|l|l|}
\hline Année & Nombre de réfugiés \\
\hline 2002 & 71 \\
\hline 2003 & 1092 \\
\hline 2004 & 3078 \\
\hline 2005 & 1173 \\
\hline 2006 & 27 \\
\hline 2007 & 0 \\
\hline 2008 & 106 \\
\hline 2009 & 818 \\
\hline 2010 & 2040 \\
\hline 2011 & 1670 \\
\hline 2012 & 630 \\
\hline Total & 10705 \\
\hline
\end{tabular}

Données fournies par le HCR, Besant Nagar, Chennai, 22 juin 2012.

Entre décembre 1987 et août 1989, 25585 réfugiés retournent à Sri Lanka par des navires affrétés par le gouvernement et le HCR. Le reste des Tamouls est retourné à Sri Lanka sans aide gouvernementale ou resté au Tamil Nadu. La deuxième guerre d'Eelam, entre Colombo et les Tigres, a débuté en juin 1990 et a abouti à la deuxième vague de réfugiés. À partir

nationalisme cinghalais, dans le cas des Tamouls de Sri Lanka ; et d'autre part face à l'hégémonie nationale des hindi-speaking dans le cas des Tamouls indiens.

${ }^{12}$ L'IPKF est une force militaire de maintien de la paix que le gouvernement de Rajiv Gandhi avait envoyée en 1987 à Sri Lanka pour s'interposer entre le LTTE et l'armée cinghalaise et favoriser un règlement du conflit. L'IPKF avait dû battre en retraite, trois ans plus tard, et Rajiv Gandhi a été assassiné par une militante du LTTE en guise de représailles. 
d'août 1989, 122000 Tamouls sri-lankais sont venus se réfugier au Tamil Nadu. Parmi eux, 115680 étaient sans ressource et ont été hébergés dans les camps. Le rapatriement de ces réfugiés a débuté le 20 Janvier 1992. Selon le HCR, du 20 janvier 1992 au 20 mars 1995, 54 188 réfugiés ont été rapatriés volontairement à Sri Lanka (Suryanarayan, 2000 ; Oberoi, 2006). La Troisième guerre d'Eelam a commencé en avril 1995 et une fois encore les réfugiés ont commencé à se diriger vers le Tamil Nadu. Ce flux de réfugiés a été la conséquence des opérations de l'armée sri-lankaise dans les zones contrôlées par le LTTE. Progressivement, le flux de réfugiés a diminué, jusqu'à l'accord de cessez-le-feu de 2002. En avril 2002, les autorités et les ONG comptabilisaient 23356 réfugiés en Inde. Pendant le cessez-le-feu, de 2002 à 2006, beaucoup d'entre eux sont retournés à Sri Lanka avec l'assistance du HCR qui fournissait des billets d'avion aux réfugiés, ou de manière illégale (à cause de l'attente pour obtenir un permis de sortie).

La quatrième guerre d'Eelam qui a commencé en 2006 et a connu une fin brutale en 2009 ne s'est pas traduite par une forte augmentation du nombre d'arrivées de réfugiés au Tamil Nadu (figure 5).

Figure 5 : Arrivées des réfugiés Tamouls sri-lankais enregistrés au camp de Mandapam de janvier 2006 au 21 mai 2010

\begin{tabular}{|l|l|l|l|l|l|l|}
\hline \multirow{2}{*}{ Période } & \multicolumn{4}{|c|}{ Adultes } & Enfants & Total \\
\cline { 2 - 7 } & Nombre de familles & Hommes & Femmes & Hommes & Femmes & Nombre de réfugiés \\
\hline 2006 & 4978 & 6343 & 5683 & 2404 & 2225 & 16665 \\
\hline 2007 & 1549 & 1639 & 1307 & 461 & 389 & 3796 \\
\hline 2008 & 1172 & 1107 & 1001 & 336 & 273 & 2717 \\
\hline 2009 & 668 & 469 & 570 & 124 & 104 & 1267 \\
\hline 2010 & 177 & 94 & 169 & 34 & 31 & 328 \\
\hline
\end{tabular}

Source : informations fournies à l'auteur par l'ONG OfERR (Chennai, juin 2010), complétées par les données fournies par Thiru Deenabandu, officier au département de réhabilitation du Tamil Nadu (Chennai, juin 2012).

Plusieurs personnes enquêtées, à l'instar d'Udaidinesh, ont eu l'expérience de ce va-et-vient entre le Tamil Nadu et Sri Lanka. Il semble que l'expérience de l'exil se dessine dans la tension entre ces deux territoires auxquels, pour aucun, l'exilé ne peut prétendre à une appartenance pleine. Cette expérience est celle du passage décrit par Serres (1997), "l'exil creuse le lieu, le vide de sa territorialité, de son assignation territoriale (qui en fait un lieu-dit) et l'ouvre à tous les possibles spatiaux " (Nuselovici, 2013 :6).

\section{Le camp, un espace de l'entre-soi ?}

Arichalmunai est le point d'entrée sur le territoire indien le plus employé par l'Eelam Shipping Service pour déposer les réfugiés provenant généralement de Talaimannar, Pesalai, Jaffna, ou encore Kilinochi. Là, les réfugiés sont soumis à un contrôle de la marine indienne, suivi d'un interrogatoire mené par les membres de la Special Branch CID (unité spéciale de la police du Tamil Nadu veillant à la sécurité intérieure) et de la $Q$ Branch CID (unité spéciale de la police du Tamil Nadu contrôlant les groupes extrémistes et terroristes). Le travail des membres de la Special et de la $Q$ Branch CID sert à faire un "premier tri " au sein des réfugiés, entre ceux qui seraient susceptibles d'être ou avoir été des membres des LTTE et 
les autres ${ }^{13}$. Les personnes soupçonnées d'avoir des liens avec le mouvement sont appelées et immédiatement écartées. Elles iront effectuer une « quarantaine » (de trente jours) dans le camp de Danuskodi avant de rejoindre le camp spécial ou special camp (SC) de Chengalpattu. Là, des agents de I'IB (Intelligence Bureau) décideront de leur sort. En août 2013 (selon les chiffres fournis lors de mes entretiens), il y avait 56 personnes enfermées dans ce camp spécial. Lors de mes terrains, je n'ai pas pu obtenir les autorisations nécessaires pour pénétrer dans ce camp. Cependant, selon l'activiste politique pro-tamoul et leader diasporique, Pala Nedumaran ${ }^{14}$ (entretiens des 6 et 8 juin 2010), il semblerait que ces chiffres soient largement sous-estimés. Selon Nedumaran, le SC de Chengalpattu ne serait pas le seul camp spécial " en fonctionnement ", il y en aurait également un à Vellore et un à Cheyyar. Cette information est confirmée par les ONG OFERR et Federation For People's Right intervenant dans les camps.

Après ces étapes, les autres réfugiés sont emmenés, généralement dans la soirée, au camp de Mandapam. Celui-ci sert normalement de camp de transit (toutefois, lors de mes enquêtes en juin 2010, on y trouvait à la fois les réfugiés arrivés après le mois d'avril et des familles installées depuis plus d'une décennie). Les jeunes hommes arrivant au camp de Mandapam sont initialement mis en quarantaine. La police de Danushkodi fait une enquête avant d'enregistrer les réfugiés et leur fournir une carte d'identité individuelle qui porte leur nom et leur adresse (nom et numéro du camp choisi, numéro de la " maison »). Une carte pour la famille est également délivrée aux réfugiés sri-lankais, qui comprend une photo de famille, les noms des membres, leur âge, les liens entre eux, leur sexe, la date et le lieu de leur arrivée en Inde, leur niveau d'instruction, de même que leur adresse à Sri Lanka. Ces cartes sont utiles lorsque les autorités doivent effectuer des vérifications d'identité à l'extérieur du camp et pour fournir les différentes aides gouvernementales.

Ainsi, à leur arrivée au Tamil Nadu, les migrants connaissent des formes variées et hétérogènes d'enfermement qui permettent de s'interroger sur les manières dont se passe leur maintien dans l'exil. Les formes que prend l'enfermement des réfugiés sri-lankais au Tamil Nadu peuvent être divisées en deux grandes catégories qui sont fonction des règles du filtrage aux frontières selon les supposées causes du déplacement. D'un côté, on trouve les ressortissants sri-lankais identifiés comme militants et enfermés dans le special camp de Chengalpattu qui s'apparente à une prison. De l'autre côté, il y a ceux qui résident dans les autres camps de réfugiés aux dispositifs matériels d'enfermement moins contraignants pour les individus qui y sont assujettis. Ces camps sont en effet des espaces ouverts mais sous contrôle, permettant ainsi une certaine liberté de déplacement à une population pauvre qui a recours au travail informel pour améliorer ses conditions de vie. Ces camps cristallisent

\footnotetext{
${ }^{13}$ Les modalités de ce tri sont fondées sur l'apparence physique des hommes. Les agents de la $Q$ Branch, avant de soumettre les réfugiés à un interrogatoire parfois long, examinent avec minutie leur corps, à la recherche de cicatrices, de blessures témoignant d'un entraînement militaire ou d'une implication au sein des LTTE.

${ }^{14}$ Pala Nedumaran est un politicien ouvertement pro-tamoul. Ancien membre du Parti du Congrès indien, il en est parti après la mort de Kamaraj pour soutenir la cause tamoule. Depuis 2002, il est le président et fondateur de la "World Tamil Confederation », depuis 1979, il préside le "Tamil National Movement », depuis 1990, il coordonne le "Tamil Eelam Liberation Supporters Co-ordination Committee » et il est l'éditeur, depuis 1997, d'un bimensuel tamoul, imprimé à Chennai et diffusé dans toute l'Asie du Sud. II a rencontré à plusieurs reprises le chef du LTTE, Velupillai Prabhakaran et entretient d'importantes relations avec les associations tamoules.
} 
également les tensions entre le gouvernement fédéral et celui du Tamil Nadu en matière de politique extérieure avec le Sri Lanka.

Abritant une population pauvre, qui a souvent recourt au travail informel, Michel Agier définit les camps de réfugiés comme des hors-lieux, des espaces de l'individu abandonné ; des espaces du bord, de la limite, des espaces d'entre-deux, où se trouve réécrite l'histoire de l'« étranger " (Agier 2011 et 2012). A l'origine conçus comme des structures temporaires, ces camps ont tous été construits pour accueillir les Indiens rapatriés de Sri Lanka. La plupart des logements dans les camps sont vétustes, car ces camps ont majoritairement été construits avant la décennie 1980 dans le but d'accueillir les quelques 300000 Tamouls indiens rapatriés de Sri Lanka dans le cadre du " pacte Sirimavo-Shastri ". Ils sont désormais inscrits dans le paysage du Tamil Nadu, représentant une situation de frontière, des espaces sous-contrôle mais liminaires. Ancrés dans le provisoire, ils ont tous été progressivement rattrapés par l'urbanisation et sont maintenant englobés dans un tissu ni totalement urbain ni totalement rural. Le camp de Keezhputhupattu est ainsi inséré entre un hôtel cinq étoiles "l'Ocean Spray " (au Nord), un resort l'hôtel "Dune " (au Sud), les eaux de l'océan indien à I'Est et la East Coast Road à l'Ouest, où se diffuse l'urbanisation de Pondichéry ${ }^{15}$. Ces camps produisent des effets, ils sont actants et génératifs de situations et de territorialités. Ils permettent de penser des processus de domination au sens d'emprise sur l'action d'autrui, mais en même temps, ces camps permettent également de négocier une autre normalité.

A ces deux dispositifs d'enfermement viennent s'ajouter les Sri Lankais qui résident hors des camps: certains Tamouls sri-lankais venus par bateau à Rameswaram ont informé les autorités qu'ils ne voulaient pas rester dans les camps de réfugiés. Ces individus sortent du processus d'encampement (Agier, 2011) parce qu'ils possèdent des moyens financiers suffisants. Néanmoins, ils doivent se faire enregistrer au poste de police le plus proche de leur lieu de résidence et obtenir un certificat de réfugié du bureau du sous-préfet (District Collector). Selon le Département de réhabilitation des réfugiés du gouvernement du Tamil Nadu, il y avait en 201031802 Tamouls sri-lankais dans cette catégorie, soit environ 30\% (entretien ONG OFERR, juin 2010 et entretien au Département de réhabilitation, juin 2010). Mais ce nombre est sous-évalué et il dépasserait les 50000 (Dasgupta, 2003 et entretien avec Suryanarayan, juin 2012). Ces réfugiés urbains vivent majoritairement dans les grandes villes de l'Etat du Tamil Nadu, en particulier à Chennai.

\footnotetext{
${ }^{15}$ Les projets immobiliers liés au développement touristique de Pondichéry font que l'urbanisation s'étend depuis une dizaine d'années le long de la ECR, à la fois vers le Nord et le Sud.
} 
Figure 6 : Distribution par âge et par sexe des réfugiés en camps

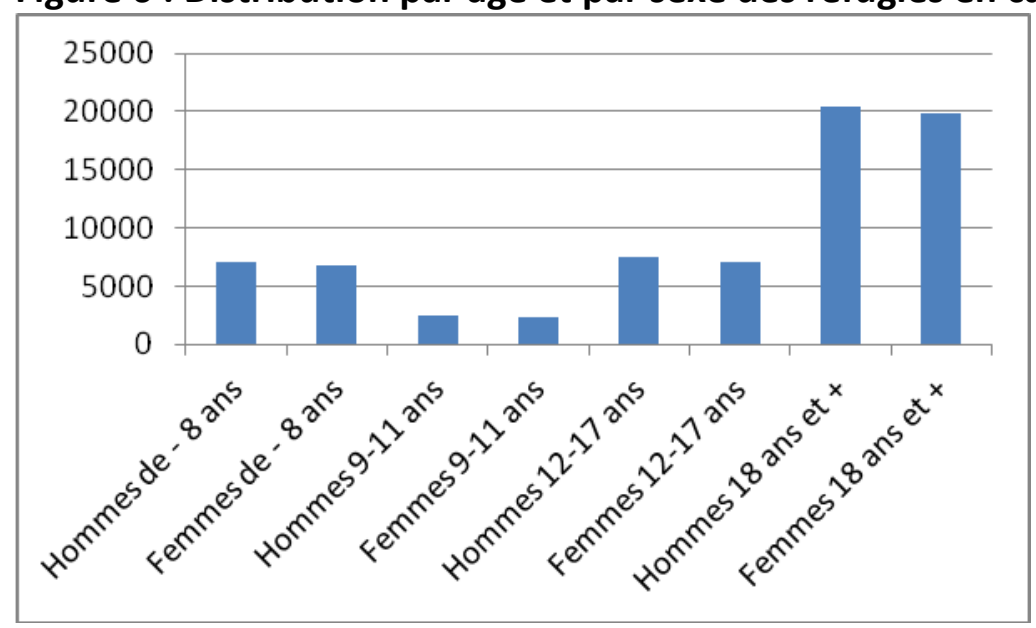

D'après les chiffres fournis par l' ONG OfERR

Ces migrants vivant dans les camps représentent un pôle important de la migration tamoule sri-lankaise dans le monde, mais ce pôle est caractérisé par son immobilité dû à l'enfermement de sa population. II semble, d'après mes entretiens, que les réfugiés présents dans les camps se considèrent comme les oubliés de la diaspora : il n'y a que peu de soutien financier de la part des organisations transnationales tamoules, seuls les réseaux familiaux assurent une modeste solidarité, ce que me confirme Samuel, membre de l'ONG OfERR ${ }^{16}$ :

" Les Tamouls de la diaspora ne donnent pas une paisâ ${ }^{17}$ pour leurs coreligionnaires vivant dans les camps. Les Tamouls de la diaspora ne participent en rien aux actions de développement que nous menons dans les camps. Nous ne connaissons pas les raisons de ce non-engagement. Nous pouvons les supposer. Ils participent au financement des associations nationalistes, entretiennent la mémoire de la guerre et surtout financent le gouvernement transnational en exil. Toutes ces actions prennent du temps et de l'argent! »

450 familles vivent dans le camp de Keezhputhupattu, représentant un total de 1726 personnes en grande majorité arrivées au cours de la décennie 1990 (Figure 7). La grande majorité de ces familles résidant au camp de Keezhputhupattu sont originaires de l'île de Mannar. Le département de réhabilitation n'exerce aucun regroupement des migrants selon leur village ou leur district d'origine. Mais en laissant la possibilité aux migrants de choisir le camp où ils veulent s'installer, on assiste peu à peu à la création de véritables figures de l'entre-soi, fruits de l'enfermement et de la contrainte.

Figure 7 : Composition de la population du camp de Keezhputhupattu en août 2013

\begin{tabular}{|l|l|l|l|l|}
\hline & $0-8$ ans & $9-11$ ans & $12-17$ ans & 18 ans et plus \\
\hline Hommes & 192 & 60 & 99 & 486 \\
\hline Femmes & 174 & 88 & 92 & 535 \\
\hline Total & 366 & 148 & 191 & 1021 \\
\hline
\end{tabular}

D’après les données recueillies au sein des ONG OfERR et FPR.

\footnotetext{
${ }^{16}$ Samuel est arrivé en Inde en 1984. Avant de rejoindre l'ONG OfERR en 1996, en tant qu'assistant administratif, il a travaillé pendant dix années dans le secteur privé. Il s'agit d'un extrait d'entretien mené le 22 juin 2012 au siège de I'ONG à Chennai.

${ }^{17}$ Centime de roupie indienne.
} 
"Depuis 23 ans je vis au Tamil Nadu et depuis 23 ans je vis dans ce camp. Même si je travaille dur, même si je gagne beaucoup d'argent, rien ne changera pour moi. Si des gens viennent te voir et te demandent d'où tu viens, tu peux dire que tu viens de France ou de Pondichéry. Moi la réponse sera je viens du camp de Keezhputhupattu. Ça marque cela quand même! Si tu ne te plais pas là où tu es tu peux t'en aller, moi ce n'est pas le cas. II n'y a pas de possibilité $d^{\prime}$ 'évolution, pas de changement. Même si on arrive à économiser 1 ou 4 lakhs ${ }^{18}$ ou un crore ${ }^{19}$, ce qui n'est vraiment pas envisageable, et bien la situation restera la même. A vie on restera là. C'est pourquoi, moi j'ai envie d'envoyer mes enfants à l'étranger. L'Australie c'est l'avenir. J'y ai envoyé mon fils aîné. Cela m'a coûté 2 lakhs. Ma cousine est déjà là-bas, c'est elle qui a arrangé le voyage. Elle connaît bien les agences, les passeurs. De toute manière partir d'ici pour l'Australie c'est assez aisé. Tu vas soit au Kerala, soit à Nagappattinam pour prendre le bateau. Pour y envoyer mon fils j'ai eu recours au cheetu, au crédit rotatif. De toute manière ici tout le monde a envie d'aller en Australie. Je dis tout le monde car on se connaît bien. Bon les gens viennent d'un peu de tout le Nord de l'île mais il y a surtout des gens de Mannar. On vit ensemble, on travaille ensemble et je peux te le dire ce que tout le monde a envie c'est de partir d'ici, et l'Australie c'est le moins cher. C'est au minimum 2 lakhs alors que pour l'Europe il faut compter au moins 8 lakhs et plus d'un crore pour Londres. J'ai de la famille en France mais c'est impossible pour moi de les rejoindre. Avant je recevais de l'argent de leur part. Mais depuis 1996 je ne reçois plus rien. Il y a eu un incendie dans le camp en 1996 et j'ai tout perdu, en particulier tous mes contacts, les numéros de téléphone, les adresses, bref tout. II faut dire que jusqu'en 1996 on vivait dans des huttes en bois et en palmes de cocotier. D'ailleurs de manière générale, les conditions de vie se sont bien améliorées. Les maisons sont en dur, on reçoit de l'aide du gouvernement et les règles au sein du camp se sont assouplies. II y a 5 ans de cela nous devions absolument rentrer tous les jours avant $18 \mathrm{~h}$ au sein du camp et il fallait signer le registre avant chaque sortie " (Entretien avec Vinesh, port de Pondichéry 2 août 2013).

A l'intérieur du camp, les abris sont organisés selon un plan orthonormé. Les allées sont étroites, en terre battue, et il n'y a pas d'éclairage extérieur. L'électricité, fournie gratuitement par le gouvernement, ne l'est que pour un usage domestique. Les abris sont simples, construits en béton et avec un toit de tôle (le sol est aussi en béton). Leur dimension est standardisée : 4 mètres par 4 ; les familles avec enfants recevant deux unités (soit une superficie totale de $32 \mathrm{~m}^{2}$ ). Les toilettes et les douches sont communes et l'eau potable est fournie par deux puits forés. Le gouvernement central de Delhi et surtout celui du Tamil Nadu accordent également des aides financières mensuelles : 1000 roupies pour le chef de famille, 750 pour sa femme et 400 pour les autres membres de la famille ${ }^{20}$. A cela $s^{\prime}$ ajoutent également des aides financières pour le mariage et la naissance des enfants ${ }^{21}$. D'ailleurs les mariages donnent lieu à des migrations entre camps. L'ensemble des personnes que j'ai interrogées sont mariées à des femmes tamoules sri-lankaises qui viennent de camps de districts autres que celui de Villupuram: il s'agit d'alliances

\footnotetext{
${ }^{18} 100000$ roupies.

${ }^{19} 1000000$ de roupies

${ }^{20}$ A cela s'ajoutent des aides spécifiques : 1000 roupies par mois pour les personnes âgées de plus de 65 ans, les veuves et les indigents. En août 2013, 1000 roupies représentaient 12,5 euros. Notons par exemple que le prix d'un kilogramme d'oignons (aliment principal de la cuisine tamoule) durant la même période était de 15 roupies.

${ }^{21}$ Le gouvernement du Tamil Nadu offre 25000 roupies pour les femmes qui se marient (50 000 roupies si la femme en question est diplômée de l'université) et offre la moitié d'un souverain d'or pour constituer le thali de la mariée. Les aides pour les naissances concernent seulement les filles, le montant de la somme est de 50000 roupies pour le premier enfant et 25000 pour le second.
} 
matrimoniales qui unissent des personnes de confessions et de castes différentes. Ces mobilités en plus de celles pour le travail sont les seules à être autorisées par le gouvernement. Les mariages mixtes sont peu nombreux. Par ailleurs, du fait que la population des moins de 18 ans soit en moyenne de $41 \%$ dans le camp de Keezhputhupattu (plus de $45 \%$ pour l'ensemble des camps) combiné au fait que ces camps sont majoritairement peuplés de jeunes adultes (les populations âgées étant restées à Sri Lanka) l'âge du mariage s'est considérablement abaissé par rapport à la norme à Sri Lanka ${ }^{22}$.

L'ensemble de ces limitations en matière de mobilité est très mal vécu par les migrants :

"II n'y a pas de problème avec la population locale, on s'entend bien. Les seuls problèmes que I'on rencontre c'est avec le gouvernement. On n'a aucune liberté de mouvement. Si l'on veut sortir du camp, il faut obtenir l'autorisation auprès de la police $Q$ Branch CID, du Revenue Inspector, du Tehsildar. C'est vraiment usant. Pourtant on n'est pas dangereux ! " (entretien avec Ruban, Port de Pondichéry, 2 août 2013).

Ces contraintes de mobilité et l'interdiction faite à toute personne de pénétrer à l'intérieur du camp sans disposer d'une permission signée par le District Collector, renforcent cet entre-soi et influent sur les pratiques des Sri Lankais. Même s'il ne s'agit pas d'un espace clos du point de vue physique (il n'y a aucun mur, aucun barbelé, aucun bornage matériel), les limites sont connues de tous, à la fois des Indiens et des migrants. Des policiers en civil assurent toutefois le bon respect des règles. Cette mise à l'écart suscite des envies de départ, des désirs d'ailleurs, souvent vers l'Australie. La grande majorité des Sri Lankais que j'ai interviewés m'ont avoué leur envie de migrer en Australie comme dans l'entretien cidessus. Les raisons de ce large plébiscite pour l'Australie ne sont pas à chercher du côté des migrants mais de ceux qui organisent le passage. L'Australie (ou plus exactement les îles Coco) est depuis 2012, la destination la moins chère et la plus aisée à atteindre par bateau en seulement 10 à 20 jours depuis les côtes du Tamil Nadu via le Sri Lanka. Selon Howie (2013), en 2012, 6412 Sri Lankais auraient fait le choix de cette route organisée avec la complicité du gouvernement de Sri Lanka.

\section{Déployer des ancrages dans les interstices de la ville}

Néanmoins, il s'agit d'une mise à l'écart qui ne l'est qu'en apparence. En effet, les initiatives des migrants infléchissent ces processus de marginalisation. Le fait que les non Sri Lankais ne puissent pas pénétrer dans le camp est jugé de manière positive. Pour les personnes interrogées, ce contrôle constitue un gage de leur sécurité. Par ailleurs à l'intérieur du camp il existe tout un tissu de services : lieux de cultes, garderie publique (ou balwadi) pour les jeunes enfants, échoppes, ateliers de mécanique, de couture.... II s'agit d'une organisation quasi-urbaine, une sorte d' " urbanité des marges " (Doraï et Puig, 2012). Cette urbanité est renforcée par l'extrême diversité des self help groups (SHGs) qui se sont constitués dans le camp et qui permettent d'entretenir des réseaux marchands. Ces réseaux participent, endedans, à la création de micro-territoires urbains, tout en étant coupés du dehors. Les habitants de ces camps créent leurs propres ressources garantes de leur survie physique et

\footnotetext{
${ }^{22}$ On assiste également à un abaissement de l'âge de la première grossesse et donc de celui du mariage. Selon Sugumaran, il y aurait beaucoup de jeunes adolescentes enceintes ce qui est marquant par rapport à Sri Lanka.
} 
morale. Précisons que la mise en place de ces ressources, témoin d'une créativité, s'opère dans certaines limites: seules les ONG exercent un rôle de médiation entre le camp et l'extérieur et peuvent alimenter ces réseaux.

"Beaucoup d'ONG interviennent dans les camps. Ce sont les seuls acteurs qui ont le droit de pénétrer dans les camps. La plus connue des ONG c'est OfERR. C'est même un gros employeur. C'est une ONG qui a été fondée par un Sri Lankais et dans laquelle ne travaillent que des réfugiés tamouls sri-lankais. Mon frère travaille au siège de l'ONG à Chennai. Mon père lui travaille sur les projets de développement de la spiruline. Mais c'est pas la seule ONG. II y a aussi ADRA (Adventist Development and Relief Agency International), PMSSS (Pondicherry Multipurpose Social Service Society) et JRS (Jesuit Refugee Service). Toutes les ONG proposent la mise en place de SHGs. Ma femme y participe. Elle fait des tissus qu'elle vend à Pondichéry». (Ruban, entretien du 16 juin 2010, camp de Keezhputhupattu).

"II y a actuellement entre 450 et 500 SHGs dans les camps. II y a des SHGs qui sont spécifiques pour les femmes. Ces SHGs sont importants pour elles. Ils sont supports d'empowerment. Par ailleurs ces SHGs sont aussi des groupes de paroles dont le rôle est salutaire, car il y a beaucoup de problèmes d'alcoolisme dans les camps et donc des problèmes de violences domestiques. [...] En matière d'emploi, le problème le plus important auquel font face les réfugiés c'est une inadéquation entre leurs compétences professionnelles et les métiers qu'ils peuvent occuper dans le but de subvenir à leurs besoins en plus de l'aide gouvernementale qui leur est offerte. Vous savez, la très grande majorité des réfugiés sont des agriculteurs ou des pêcheurs. Ils doivent donc s'adapter au marché du travail, acquérir de nouvelles qualifications. Ils ont du acquérir entre 10 et 15 compétences différentes depuis leur arrivée. La grande majorité de ces compétences ne leur seront pas utiles s'ils retournent à Sri Lanka. C'est par exemple toutes les compétences apprises par les hommes ou les femmes dans la construction, la peinture, la mécanique...C'est pourquoi nous avons pensé à leur offrir de nouvelles formations pouvant développer des compétences utiles lors de leur retour, comme l'informatique » (entretien avec Samuel, 22 juin 2012, ONG OfERR, Chennai).

Les SHGs mis en place par les ONG s'adressent majoritairement aux femmes. La part des femmes dans les camps au Tamil Nadu était en 2013 de 49,2 \%. II y a au sein du camp de Keezhputhupattu une équi-répartition entre hommes et femmes. Cette participation au SHGs leur permet d'acquérir une autonomie, car pour la grande majorité des femmes mariées, leur conjoint ne les autorise pas à travailler en dehors du camp. II y a pourtant différentes opportunités de travail qui s'offrent à elles, bien que ces emplois soient majoritairement peu qualifiés et peu rémunérés, particulièrement dans les secteurs de la construction et de l'agriculture. Les quelques femmes sri-lankaises qui travaillent en dehors du camp de Keezhputhupattu, sont veuves ou sont, pour un temps, à la tête du ménage. Dès lors ce travail est une nécessité qui vient compléter une aide gouvernementale jugée insuffisante. Les difficultés que peuvent rencontrer les femmes sont résumées par Hans de la façon suivante :

"The Sri Lankan refugee woman, the conservator of the cultural identity of the family, is confronted with hard choices in refuge. This is more so in Tamil Nadu, where the caste structures are rigidly stratified, and gender roles are defined by caste and not by class. As long as the refugees stays in India and does not want to be a figure of ridicule by the local 
Tamils, she has to follow similar practices. This creates further stress in a life already traumatized by war, violence and uprooting "(Hans, 1997: 7) ${ }^{23}$.

La visibilité des réfugiés sri-lankais dans l'espace public de Pondichéry résulte de deux types de mobilités. La première, qualifiée de non contrainte définit des mobilités privées. Ces mobilités privées, sont largement encouragées par les ONG et les SHGs mais limitées par les le processus d'encampement qui fait du camp l'espace d'une altérité politique et culturelle. Ces mobilités privées concernent surtout la pratique religieuse. La deuxième qualifiée de contrainte, concerne les mobilités professionnelles effectuées dans le cadre de l'emploi. Elles sont les plus nombreuses. Elles évoluent en fonction de l'offre d'emploi et de la capacité des ONG et des associations et fondations religieuses (ADRA, JRS, PMSS) à développer de nouvelles formations. Par exemple, la fondation JRS développe exclusivement des formations de couture pour les femmes quand en même temps I'ONG OfERR développe des formations en informatique. Si les mobilités des réfugiés Sri Lankais sont contrôlées, celles-ci génèrent des formes de cohabitation inédites en se déployant dans les interstices de la ville telles que le port de Pondichéry, le débit de boisson d'arack, ou encore les espaces situés sur les fronts d'urbanisation dans lesquels les Sri Lankais travaillent comme manutentionnaires dans la construction, ou dans les ateliers de couture. Les mobilités liées à l'emploi sont celles qui engendrent de la coprésence entre réfugiés et Indiens.

"Tous les jours, après le travail je dois retourner au camp, et une fois par semaine je dois rester toute la journée au sein du camp. Je ne peux pas aller ailleurs. Ce sont les règles, il n'y a pas d'autres choix. Pour se rendre au travail c'est l'employeur qui fait apprêter des véhicules pour venir nous chercher. J'ai mes habitudes et avec mes amis on monte dans le même véhicule. Quand c'est la pêche on se rend au port sinon on va travailler sur les chantiers de construction de nouveaux bâtiments. Je suis peintre, charpentier et pêcheur. C'est durant la journée de travail que l'on côtoie la population tamoule. Au retour généralement on s'arrête boire de l'arack et parfois on achète des bières. On les boit hors du camp, près du stade, il y a une grande friche seulement peuplée de chèvres. C'est là où tout le monde vient boire ". (entretien mené avec Ruban, 2 août 2013, port de Pondichéry).

C'est particulièrement vrai durant la saison de pêche. L'employeur de ces 731 réfugiés est un tamoul indien de Pondichéry. II profite bien évidemment de cette main d'œuvre peu chère, puisque les réfugiés gagnent en moyenne 1500 roupies par semaine pour un travail harassant de manutention. Au port, tout est codifié. Les pêcheurs indiens sont vêtus d'un vêtement de même couleur. Une fois qu'ils ont accosté, ils attendent que le même employeur des réfugiés négocie les prix des poissons. Après, le défilé commence : les réfugiés entrent en scène à l'aide de caisses de différentes couleurs (selon l'acheteur) pour décharger la cargaison puis l'embarquer dans des camions. Dans une journée de 14 heures, entre ces moments d'intenses activités, il y a aussi de longues pauses où les réfugiés en profitent pour échanger, discuter entre eux et avec les pêcheurs.

\footnotetext{
${ }^{23}$ Les femmes sri-lankaises réfugiées, considérées comme les garantes de l'identité culturelle et des valeurs familiales sont confrontées à des choix difficiles en situation d'asile. C'est d'autant plus prononcé dans le cas du Tamil Nadu, où les structures de caste sont rigidement stratifiées, et les rôles de genre définis par la caste et non par la classe. Tant que ces femmes séjournent en Inde, elles doivent adopter des pratiques similaires à celles des tamoules si elles ne veulent pas être tournées en ridicule. Cela crée un stress supplémentaire dans une vie déjà affectée par la guerre, la violence et le déracinement (Hans, 1997 : 7).
} 
Néanmoins, même si ces moments permettent aux réfugiés d'accéder à des formes de citoyenneté, la majeure partie d'entre eux se sentent aliénés.

" Je me sens complètement aliéné. Je ne suis plus sri-lankais et je ne serai jamais indien. Je vis avec ma femme et mes enfants dans le camp de Keezhputhupattu, mais il n'y a pas de bonheur. De toute manière, tout le monde nous traite comme des sujets secondaires, des sujets subalternes. Tout le monde se moque de nous, surtout les politiciens. Cela fait maintenant 23 ans que nous réclamons la citoyenneté indienne. On sait qu'on ne l'aura jamais, mais c'est important. Ici je suis satisfait pour ma sécurité et parce que d'une certaine manière je renoue avec mon ancienne activité. Comme beaucoup de personnes dans le camp, j'étais pêcheur. Travailler au port, même si c'est pour casser de la glace, débarquer les bateaux, et remplir des caisses de poissons, cela me rappelle un peu mon ancienne activité et donc mon chez-moi. En plus parfois les pêcheurs nous laissent utiliser leurs bateaux dans les moments d'attente. Ma femme aussi pêchait à Sri Lanka. Quand j'étais à Mannar, je gagnais bien ma vie, c'était suffisant pour l'ensemble de ma famille. Ici ce n'est pas le cas, je gagne au maximum 1500 roupies par semaine. Mais tout cela ne dure qu'un temps. Après la pêche, il va falloir trouver un autre emploi. C'est la saison pour 5 mois, mais après il faut trouver à s'employer dans la construction, toute sorte de travail de coolie. Moi je suis un travailleur qualifié, mais je dois exécuter ce que les autres ne veulent pas faire. On n'a pas le choix, il faut survivre. On est comme des esclaves. Bon si je voulais je pourrai retourner à Sri Lanka puis revenir ici. Mais à mon retour je n'aurai pas la permission de la part de l'Etat de vivre dans un camp. Et là je n'aurai plus d'aides ». (Entretien mené avec Ruban, port de Pondichéry, 2 août 2013).

Figure 8 : Travailleurs Sri Lankais au port de Pondichéry

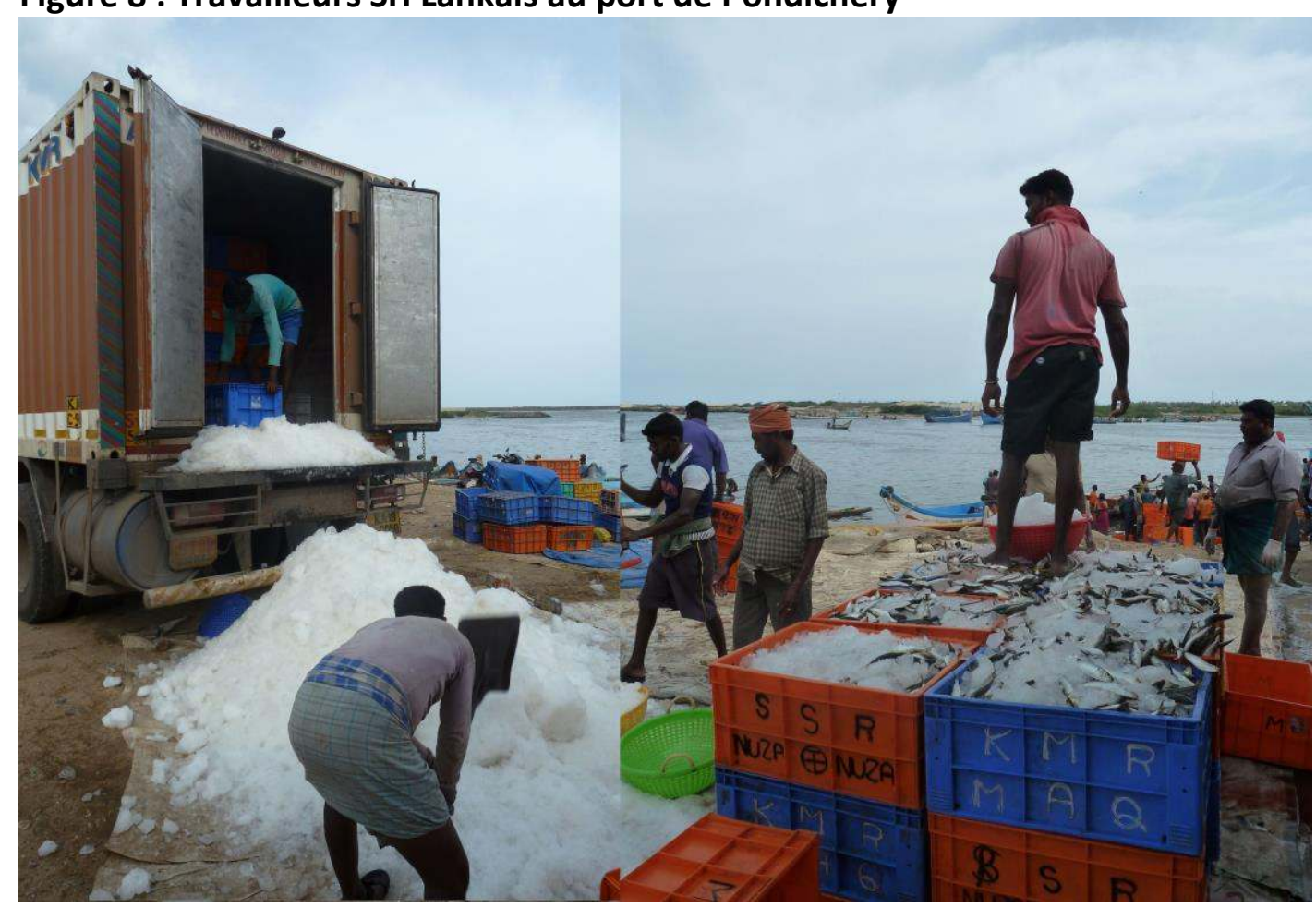

Clichés : Goreau-Ponceaud, août 2013. De dos sur la photo de droite : Ruban.

Ce sentiment d'aliénation est d'autant plus fort que les emplois pourvus par les réfugiés sont difficiles, peu qualifiés et peu payés. Ces emplois échappent également au secteur formel. 
Dans tous mes entretiens un mot revenait sans cesse dans la bouche de ces réfugiés pour désigner leur situation : adimai (littéralement esclaves). Ces travailleurs ont le sentiment d'être dépossédés, de subir une négation de leur individualité. Cette aliénation est l'expression d'une double distance : de la réalité du lieu d'origine et de la réalité du lieu d'accueil. La tension générée par l'écart entre l'Inde et le Sri Lanka produit une expérience particulière : l'exilé est celui qui ne cesse d'expérimenter, sans que cela ne se sédimente en matériau culturellement transmissible, puisque la culture d'origine ne peut dire celle d'arrivée et vice-versa (Nuselovici, 2013). Cette dualité de l'ancrage et du mouvement met le réfugié dans une éternelle et inconfortable position d'intermédiaire : entre le formel et l'informel, entre les pêcheurs et les cols blancs du secteur, entre ici et là-bas, entre dedans et dehors, entre inclus et exclus. Dès lors ces espaces-écarts à l'instar des camps ou du port de Pondichéry sont des espaces transitionnels permettant le passage graduel de l'un à l'autre, des espaces de contact et de rupture à la fois.

\section{Conclusion}

Initialement installés à la marge des villes, les camps des migrants sri-lankais ayant fui la guerre ont été pour une grande partie rattrapés par l'urbanisation, tel est le cas du camp de Keezhputhupattu situé au Tamil Nadu, à la frontière du territoire de Pondichéry. Sans être intégrés dans le tissu urbain, ces réfugiés ne sont pas entièrement dépendants de l'aide mise en place par le département de réhabilitation du Tamil Nadu. Dans cet espace écart s'est développée une urbanité des marges qui témoigne des pratiques et des initiatives des migrants pour infléchir les processus de marginalisation. Ce camp est simultanément un espace de rejet de la part de la société indienne et un espace sécurisant où les réfugiés srilankais retrouvent et reconstituent à des degrés divers une communauté locale. Même si leurs mobilités sont réduites et contrôlées, les déplacements quotidiens pour le travail permettent à ces migrants au statut incertain, d'occuper l'espace public. Cette coprésence permise par le travail pose le thème de la reconnaissance de ces réfugiés. Reconnaissance qui ne peut se faire sans l'intersubjectivité et la différence, d'où un entrelacement de la figure du non-citoyen et du citoyen, du migrant et de "l'établi ". Néanmoins, il subsiste un malaise important qui se traduit à chaque fois dans les propos recueillis auprès de ces migrants: le sentiment d'éprouver un manque, une absence. Comme si l'expérience de l'exil, née de la traversée du détroit de Palk, avait marqué le corps déplacé d'une déchirure avant de devenir une condition. Il se développe et se construit à l'intérieur de ces espaces de l'entre-deux des processus sociaux, des configurations spécifiques d'idées et de valeurs au sein d'un monde où la marginalité est généralisée. Avec leurs différentes manières de faire, de prendre place, les réfugiés sri-lankais créent leur propres ressources, cette créativité est garante d'une autre normalité, fragilisée cependant par leur position d'intermédiaire. Ces réfugiés demeurent pauvres et exercent souvent des activités dans l'économie informelle et ne parviennent pas à se libérer des effets de la stigmatisation (liés à l'encampement et à leur statut).

Réclamant depuis leur arrivée une loi nationale et une citoyenneté indienne, l'individu réfugié, détaché de ses contextes de socialisation et d'identification (laissés aux lieux de la " personne ") est stoppé dans un écart, en reste, selon une place assignée en dehors de tout espace de reconnaissance politique (Agier, 2012 : 66). 


\section{Bibliographie}

AGIER M. (2012) «Penser le sujet, observer la frontière. Le décentrement de I'anthropologie ", L'Homme, 2012/3, n²03-204, pp. 51-75.

AGIER M. (2011), Le couloir des exilés. Etre étranger dans un monde commun, Paris, Editions du croquant, 120p.

BAUJARD J. (2012) "Etre réfugié à Delhi : politiques d'asile et trajectoires des réfugiés ", Emigrinter, 2012/8, pp.6-25.

BAUMAN Z. (2010), Identité, Paris, Editions de l'Herne, 136p.

BRUBAKER R. (2005) "The 'diaspora' diaspora », Ethnic and Racial Studies, 2005/ 28, pp. 119.

DASGUPTA A. (2003) "Repatriation of Sri Lankan Refugees. Unfinished Tasks", Economic and Political Weekly, 2003/28, n²4, pp.2365-2370.

DORAII K et PUIG N. (2012), Un lointain si proche. L'urbanité des marges. Migrants et réfugiés dans les villes du Proche-Orient, Paris, Editions Téraèdre/IFPO, 335p.

GOREAU-PONCEAUD A. (2014) "Ganesha Chaturthi and the Sri Lankan Tamil Diaspora in Paris: inventing strategies of visibility and legitimacy in a plural 'mono-cultural' society", in Gallo E., Migration and Religion in Europe. Comparative perspectives on South Asian experiences, Farnham, Editions Ashgate, pp. 211-231

GOREAU-PONCEAUD A. (2012) "Routes et antiroutes de l'immigration tamoule srilankaise », E-migrinter, 2012/8, pp.26-44.

GOREAU-PONCEAUD A. (2011) « L'immigration sri-lankaise en France : Trajectoires, contours et perspectives », Homme et Migrations, 2011/1291, pp.26-39.

GOREAU-PONCEAUD A. (2008), La diaspora tamoule: trajectoires spatio-temporelles et inscriptions territoriales en Île-de-France, Thèse de géographie non-publiée, Université de Bordeaux 3, 427p.

HANS A. (1997) “Sri Lankan Tamil refugee women in India”, Refuge, 16/2, pp.3-9.

HOWIE E. (2013) "Sri Lankan boat migration to Australia. Motivations and dilemma", Economic and Political Weekly, 2013/48, n³1, pp. 97-104.

JULLIEN F. (2012) « L'écart et l'entre. Ou comment penser l'altérité », FMSH-WP, 2012/3.

LUSSAULT M. (2009), De la lutte des classes à la lutte des places, Paris, Editions Grasset, $220 p$. 
MEYER E. (2001), Sri Lanka, entre particularismes et mondialisation, Paris, Editions La Documentation Française, 184p.

NUSELOVICI (NOUSS) A. (2013) «L'exil comme expérience », FMSH-WP, 2013/43.

OBEROI P. (2006), Exile and Belonging. Refugees and State Policy in South Asia, New Delhi, Editions Oxford University Press, 298p.

SAID E. (2008), Réflexions sur l'exil et autres essais, Arles, Editions Actes Sud, 768p.

SAMADDAR R. (2003), Refugees and the State. Practices of Asylum and care in India, 19472000, New Delhi, Editions Sage, 499p.

SEN S. (2003) "Paradoxes of the international regime of care: the role of the UNHCR in India", in Samaddar R., Refugees and the State. Practices of Asylum and care in India, 19472000, New Delhi, Editions Sage, pp. 396-442.

SERRES M. (1997), Atlas, Paris, Editions Champs Flammarion, 279p.

SINGH D. (2003), Indian Peacekeeping Force in Sri Lanka 1987-1989, Delhi, Editions Natraj Publishers, 206p.

SMITH C. (2008) " Les LTTE, mouvement de libération et d'oppression nationale ", In GAYER L., JAFFRELOT C., Milices armées d'Asie du Sud, Paris, Editions Sciences Po, pp. 95-121.

SURYANARAYAN V. (2000), Between fear and hope: Sri Lankan refugees in Tamil Nadu, Chennai, Editions TR Publications, 116p. 